\title{
Chronopharmacology: A New Approach for Drug Delivery Schedules
}

P D Gupta

Former Director Grade Scientist Centre for Cellular and Molecular Biology, Hyderabad. India

Corresponding Author: P D Gupta, Former Director Grade Scientist Centre for Cellular and Molecular Biology, Hyderabad. India.

Received date: January 30 2021; Accepted date: February 19, 2021; Published date: February 23,2021

Citation: P D Gupta. (2021) Chronopharmacology: A New Approach for Drug Delivery Schedules. Clinical Research and Clinical Trials. 3(1); DOI: $10.31579 / 2693-4779 / 022$

Copyright: (c) 2021, P D Gupta. This is an open access article distributed under the Creative Commons Attribution License, which permits unrestricted use, distribution, and reproduction in any medium, provided the original work is properly cited.

\begin{abstract}
The discovery of biological clock has changed many established concepts and hypotheses in pharmacology and medicine. Diseases borne due to disturbances or deregulation of biological clock are set right only by setting the clock right. There are many natural and life style factors which deregulate the clock. Recently many chronotherapeutic drugs were discovered and tested this review throw light on such problems.
\end{abstract}

Keywords: chronopharmacology; drug delivery schedules; pharmacology; chronotherapeutic drugs

\section{Introduction:}

Rhythm is life, without it life does not exist. Heart beat and respiration are rhythmic and these rhythms sustain life. The continuation of life is by reproduction the only biological function of our body is to reproduce; the other functions are evolved to help the reproduction Rhythm is defined as "Regular recurrence of quantities or accents of an event". Reproduction is rhythmic and if it becomes non-rhythmic, individuals cannot reproduce (1-4). Until puberty, reproductive rhythm does not exist in males or females and therefore, they are not able to reproduce (5-7). In adults estrogen receptor signalling modulates circadian rhythms in females.

A new study has found that chronic disruption of one of the most basic circadian (daily) rhythms -- the day/night cycle -- leads to weight gain, impulsivity, slower thinking, and other physiological and behavioural changes in mice, similar to those observed in people who experience shift work or jet lag.(2)

Human health and healthy ageing are related to circadian rhythms; more so the disruption is bidirectional. Circadian dysfunction can either result from abnormal timing or decreased amplitude of circadian signals. This dysfunction is associated with an increased risk for neurodegenerative disease, cardiometabolic disease and malignancy. Many seizures occur in diurnal, sleep/wake, circadian, or even monthly patterns. The relationship between biomarkers and state changes is still being investigated, but early results suggest that some of these patterns may be related to endogenous circadian patterns whereas others may be related to wakefulness and sleep or both. Chronotherapy, the application of treatment at times of greatest seizure susceptibility, is a technique that may optimize seizure control in selected patients (8). It may be used in the form of differential dosing, as preparations designed to deliver sustained or pulsatile drug delivery or in the form of 'zeitgebers' that shift endogenous rhythms. Early trials in epilepsy suggest that chronopharmacology may provide improved seizure control compared with conventional treatment in some patients. (8)

\section{Rhythmic Notes}

What sounds like music to our ears is actually a synchronized combination of musical notes, much like the way hormones produced by endocrine glands act in harmony to regulate the rhythm of reproduction. The notes in the reproductive rhythm are the glands - pineal, hypothalamus, pituitary and testis or ovary - which produce various hormones that interact in a specific and sequential manner to regulate this vital body function (9)

\section{Factors influencing the rhythms:}

The nodes in the rhythm of reproduction are affected by environmental factors such as light, temperature, altitude, radiation, etc. (2). And the physiological factors such as aging, diseases and heredity. These factors control the production of key hormones in the endocrine glands. For example, the production of melatonin in pineal gland is controlled by light, which in turn controls sex cycle in females and males.

\section{Aging and the clock}

Aging and low levels of daytime light exposure lead to gradual attenuation and disruption of circadian rhythms. Both aging and circadian rhythm disruption increase the risk of Alzheimer's disease (AD), further disrupting of circadian rhythms. Disruption of circadian rhythms, notably the circadian sleep-wake rhythm, increases AD neuropathological changes such as brain amyloid-beta accumulation (10). The mechanisms underlying the viscous cycle of disrupted circadian rhythms and progression of $\mathrm{AD}$, as well as the normal age-related circadian rhythm alterations, and are described in this article.

\section{Consequences in shift workers}

Shift work inevitably leads to the displacement of rest-activity, sleepwake and fasting-feeding cycles. In turn, this may cause misalignment of 
the endogenous circadian timing system with the external environment as well as sleep disturbance (Fig.1). While accumulating evidence suggests that this has negative effects on cardiovascular and metabolic health, more research is required for the development and implementation of strategies that prevent or mitigate the adverse health effects of shift work(2).

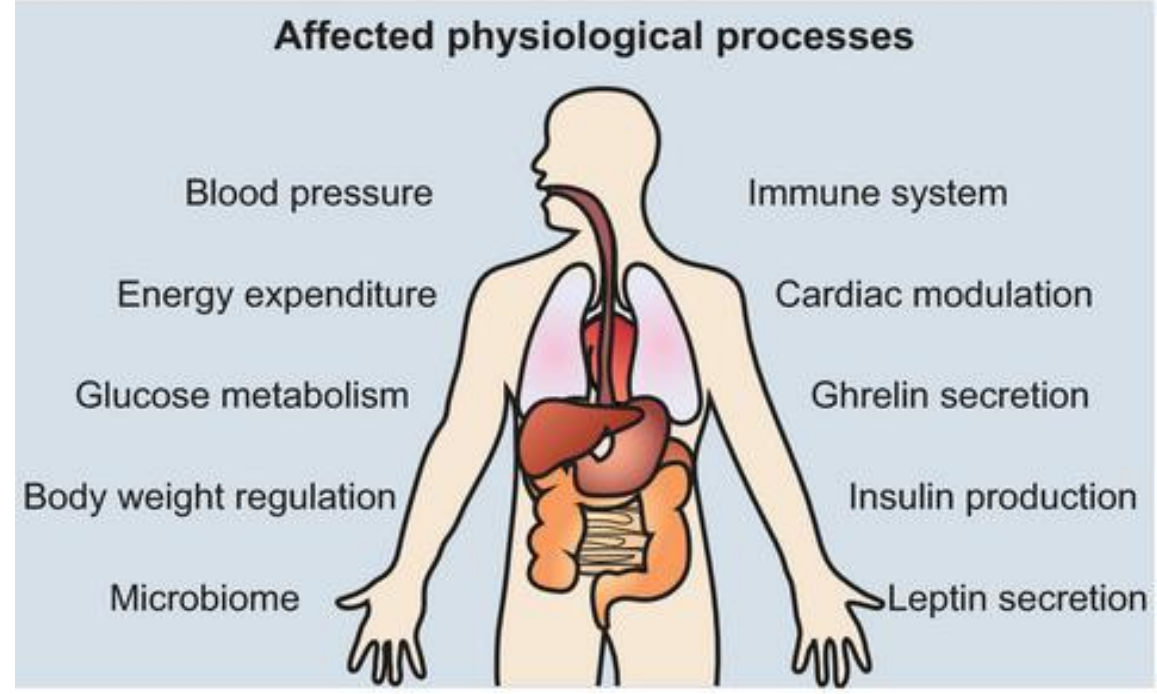

Fig 1 Affected physiological processes due to working in night.

\section{Chronotherapeutics}

Circadian rhythm also regulates many other functions in humans, such as, metabolism, physiology, behaviour, sleep patterns, hormone production, etc. the kinetics \& dynamics of medication directly affected by endogenous biological rhythm $\&$ also how the dosing time of medications affects biological timekeeping \& features ( period, level, amplitude \& phase ) of biological rhythms. The circadian rhythm disorder can be longterm and caused by internal factors such as age, genes, or a medical condition. Symptoms may include extreme daytime sleepiness, insomnia, tiredness, decreased alertness, and problems with memory and decisionmaking. These disorders can easily managed by Chronopharmacology, which is comparatively new branch in pharmacology.

Chronopharmacotherapy, chronopharmacokinetics \& Chronotoxity are the sub branches of chronopharmcology.. Chronopharmacotherapy is the investigative science that elucidates the biological rhythm dependencies of medication. Chronopharmaceutics is a branch of pharmaceutics (science and technology of drug dosage forms) meant to the design and evaluation of drug delivery systems that release a bioactive agent at a rhythm that ideally matches in real time the biological requirement for a given disease therapy or prevention uses the basic concepts of human chrono biology and the rhythm dependence of certain disease states and the pharmaco dynamics of medications (11). It is useful to solve problems of drug optimization i.e. to enhance the desired efficiency or to reduce its undesired effects. Chronopharmacologic approaches involve a lesser risk of errors and or false information than the conventional homeostatic approach. The effectiveness \& toxicity of many drugs vary depending on dosing time associated with 24 hours rhythm of biochemical, physiological \& behavioural process under the control of circadian clock such chronopharmacological phenomenon are influenced by not only the pharmacokinetics but also pharmacodynamics of medication. Now a day Chronopharma- cological principle are used in the therapy of various cardiovascular diseases such as hypertension, myocardial infarction, angina pectoris, pulmonary embolism etc. blood pressure fluctuates according to the circadian pattern. Continuous monitoring of blood pressure throughout the day and night reveals a pattern with minimum values of systolic \& diastolic pressure between midnight \& 4 am. Early in the morning B.P begins to rise from the low levels reached during sleep.
Increases in blood pressure are accompanied by increase in heart rate caused by the chemical generated by the body \& delivered into the blood stream.

The drug therapy can be optimized by tailoring the dosing schedule based on chrono- biological pattern. The safety and efficacy of the drug is achieved by coordinating the peak plasma concentration of the drug with circadian rhythm of the body

\section{Experiments with blind}

Most blind people with no perception of light, however, experience continual circadian desynchrony through a failure of light information to reach the hypothalamic circadian clock, resulting in cyclical episodes of poor sleep and daytime dysfunction (12). They have constantly high levels of melatonin in their blood, since serotonin is converted in to melatonin in the dark. The safe and effective medicine for this disorder include melatonin and the melatonin agonist Tasimelteon, however doses have to be standardised (13).

Gut microbiota can influence the host's circadian gene expression through direct interactions via immunoreceptors and microbiota-derived metabolites, especially in peripheral tissues. Noteworthy metabolites that are only attributable to the microbiota are short-chain fatty acids and unconjugated bile acids. The microbiota also serves as a mediator for the interplay between the host's diet and circadian rhythmicity (14). This work furthermore displays that the microbiota is subject to diurnal variations in terms of structure and function and that the host and the host's diet influence these fluctuations.

\section{Drug Delivery}

The release of some drugs is preferred in pulses i.e. the release of drug as a "pulse" after a lag time has to be designed in such a way that a complete and rapid drug release should follow the lag time. (15) Lag time is defined as the time between when a dosage form is placed into an aqueous environment and the time at which the active ingredient begins to release from the dosage form. These systems are also called time-controlled as the drug released is independent of the environment $(16,17)$. A single dosage form provides an initial dose of drug followed by one release-free interval, after which second dose of drug is released, which is followed 
by additional release-free interval and pulse of drug release. Some drugs, such as beta blockers, and salicylamide, undergo extensive first pass metabolism and require fast drug input to saturate metabolizing enzymes in order to minimize pre-systemic metabolism (18) thus, a constant/sustained oral method of delivery would result in reduced oral bioavailability.

\section{Conclusions}

Earlier it was difficult to treat the diseases borne due to deregulation of biological clock. These diseases were out of the purview of pharmacology. However after the understanding of this clock it was possible to formulate some drugs through the study of chronopharmacodynamics of some chemical compounds and manage such diseases so as to get some relief the patients.

\section{References}

1. Gupta, P.D. and Pushkala, K. (2012) Clocks within us LAMBERT Academic Publishing, GmbH \& Co. KG, Saarbrücken, Germany.

2. Pushkala, K. and Gupta,P.D. (2011) Dark side of the night light (Monograph) LAMBERT Academic Publishing, GmbH \& Co. KG, Saarbrücken, Germany.

3. Seán T. Anderson, Garret A. (2020) FitzGerald Sexual dimorphism in body clocks Science 369, (6508)1164-1165.

4. Gupta, P.D. and Pushkala, K. J Anall Oncol, (2016), 146-152.

5. Gupta P D and Iino A Mothering A Cause. Oxford \& IBH Publishers New Delhi
6. Gupta PD and Pushkala K Human Genet \& Embryol (2018), 1

7. PD Gupta and K Pushkala. (2020) Clinical J Ophthalmology and Eye Care Review. 2(1).

8. Gupta P D in Concepts in Chronopharmacology. ed N. (2009) Udupa and P D Gupta, Shyam Prakashan, Jaipur.

9. Gupta P D Hormone Harmony, Publication Division CSIR, New Delhi, 1996.

10. https://www.sleep.theclinics.com/article/S1556-407X(15)00101$0 / a b s t r a c t$

11. Kuila, A., N. V. Dhandapani, H. Bhowmik, A. Soni, and K. H. Kumar. Asian J Pharm Clin Res. 11, (8), 15-20, [ 2018]

12. Steven W. Lockley, Josephine Arendt Debra J. Skene, Dialogues Clin Neurosci. 9(3): 301-314. [2007]

13. S W Lockley, M A Dressman et al.. Lancet P1754-1764, [ 2015]

14. Christoph A Thaiss, Maayan Levy et al. Cell;167(6):14951510.e12. [2016]

15. Gupta A in Concepts in Chronopharmacology. ed N. (2009) Udupa and P D Gupta, Shyam Prakashan, Jiapur.

16. Usha YogendraNayaka Gopal Venktesh et al. J Controlled Release 136, (2), , 125-131 [2009]

17. Usha Yogendra Nayak, Gopal Venktesh Shavi,et al J Controlled Release136(2) 125-131 [2009]

18. (https://www.sciencedirect.com/topics/pharmacologytoxicology-and-pharmaceutical-science/salicylamide [2018].
This work is licensed under Creative Commons Attribution 4.0 License

To Submit Your Article Click Here: Submit Article

DOI: $10.31579 / 2693-4779 / 022$
Ready to submit your research? Choose Auctores and benefit from:

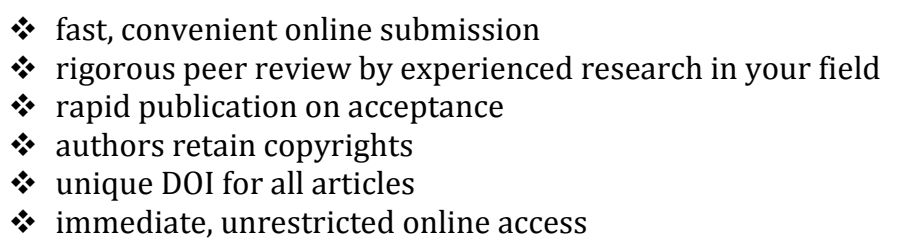

At Auctores, research is always in progress.

Learn more www.auctoresonline.org/journals/clinical-research-andclinical-trials 